\title{
SYSTEMIC MESENCHYMAL STEM CELL ADMINISTRATION ENHANCES BONE FORMATION IN FRACTURE REPAIR BUT NOT LOAD-INDUCED BONE FORMATION
}

\author{
A.E. Rapp ${ }^{1}$, R. Bindl ${ }^{1}$, A. Heilmann ${ }^{1}$, A. Erbacher ${ }^{2}$, I. Müller ${ }^{3}$, R.E. Brenner ${ }^{4}$ and A. Ignatius ${ }^{1}$ \\ ${ }^{1}$ Institute of Orthopaedic Research and Biomechanics, Centre of Musculoskeletal Research, University of Ulm, \\ Germany \\ ${ }^{2}$ Department of General Paediatrics, Haematology and Oncology, University Children's Hospital Tübingen, \\ Tübingen, Germany \\ ${ }^{3}$ Clinic for Paediatric Haematology and Oncology, Bone Marrow Transplantation Unit, \\ University Medical Centre Hamburg-Eppendorf, Germany \\ ${ }^{4}$ Department of Orthopaedics, Division of Joint and Connective Tissue Diseases, \\ Centre of Musculoskeletal Research, University of Ulm, Germany
}

\begin{abstract}
Mesenchymal stem cells (MSC) were shown to support bone regeneration, when they were locally transplanted into poorly healing fractures. The benefit of systemic MSC transplantation is currently less evident. There is consensus that systemically applied MSC are recruited to the site of injury, but it is debated whether they actually support bone formation. Furthermore, the question arises as to whether circulating MSC are recruited only in case of injury or whether they also participate in mechanically induced bone formation.

To answer these questions we injected green fluorescent protein (GFP)-labelled MSC into C57BL/6J mice, which were subjected either to a femur osteotomy or to noninvasive mechanical ulna loading to induce bone formation. We detected GFP-labelled MSC in the early (day 10) and late fracture callus (day 21) by immunohistochemistry. Stromal cell-derived factor 1 (SDF-1 or CXCL-12), a key chemokine for stem cell attraction, was strongly expressed by virtually all cells near the osteotomy - indicating that SDF-1 may mediate cell migration to the site of injury. We found no differences in SDF-1 expression between the groups. Micro-computed tomography $(\mu \mathrm{CT})$ revealed significantly more bone in the callus of the MSC treated mice compared to untreated controls. The bending stiffness of callus was not significantly altered after MSCapplication. In contrast, we failed to detect GFP-labelled MSC in the ulna after non-invasive mechanical loading. Histomorphometry and $\mu \mathrm{CT}$ revealed a significant loadinduced increase in bone formation; however, no further increase was found after MSC administration. Concluding, our results suggest that systemically administered MSC are recruited and support bone formation only in case of injury but not in mechanically induced bone formation.
\end{abstract}

Keywords: mesenchymal stem cells, cell therapy, bone formation, fracture healing, mechanical loading, mouse model.

\section{Corresponding author:}

Anna E. Rapp

Institute of Orthopaedic Research and Biomechanics Helmholtzstr. 14, 89081 Ulm, Germany

Phone: +49-731-500-55340

Fax: +49-731-500-55302

E-mail: anna.rapp@uni-ulm.de
Mesenchymal stem cells (MSC), also termed mesenchymal stromal or progenitors cells, are a promising tool in regenerative therapies because of their great potential for proliferation and differentiation as well as their ability to secrete a broad spectrum of biologically active factors with paracrine regenerative and anti-inflammatory effects (Caplan, 2007). MSC and other progenitor cells types like endothelial progenitor cells have supported bone regeneration in animal experiments (Atesok et al., 2010; Chandrasekhar et al., 2011; Shoji et al., 2010) and in clinical cases (Hernigou et al., 2005), when they were locally transplanted into bone defects and poorly or non-healing fractures. The benefit of systemic MSC transplantation to improve bone healing is currently less evident.

Bone healing is a complex and highly regulated process, with consecutive and closely linked phases of inflammation, repair and remodelling (Claes et al., 2012). The early inflammatory phase of fracture healing, which is characterised by a complex interaction between immune cells, resident tissue cells and pro- and antiinflammatory cytokines and chemokines, is thought to initiate the repair cascade by attracting mesenchymal progenitor cells locally, including from the periosteum (Colnot et al., 2012) and the bone marrow (Taguchi et al., 2005 ). There is evidence suggesting that circulating MSC are physiologically recruited to the fracture site and contribute to osteogenesis (Kumagai et al., 2008), implying that increasing their numbers in the blood circulation by intravenous administration may support fracture repair. However, the experimental results are varied (Devine et al., 2002; Granero-Molto et al., 2009; Lee et al., 2009b). There is consensus, that systemically applied MSC are recruited to the fracture site (Devine et al., 2002; Granero-Molto et al., 2009; Lee et al., 2009b). Although the underlying mechanisms are not fully elucidated, several cytokines/ chemokines present during the early phase of fracture healing are known to be strong chemoattractants for MSC; including, platelet-derived growth factor (PDGF), the hypoxia regulated CXC-motif chemokine 12 (CXCL-12, also termed stromal cell derived factor (SDF)-1), the C-C motif ligand 2 chemokine (CCL2) and the macrophage chemotactic protein (MCP-1) (Fiedler et al., 2004; Granero-Molto et al., 2009). 
However, it is debated whether systemically applied MSC, once they are recruited, actually support fracture repair. While Granero-Moltó et al. reported that intravenously injected MSC induced the formation of a larger callus with greater amounts of newly formed cartilage and bone (Granero-Molto et al., 2009), other authors did not find improved healing - although they confirmed the recruitment of the applied cells to the bone injury site (Devine et al., 2002).

Furthermore, the question arises as to whether circulating MSC are recruited only in fracture healing initiated by an inflammatory reaction or whether they also participate in mechanically induced bone formation, which is not associated with inflammation. Mechanical loading is a known potent stimulus for bone formation (Lee et al., 2002) and is, therefore, used in therapeutic strategies for the treatment of osteopenic disorders, including osteoporosis (Gusi et al., 2006). Differential gene analysis in loaded and non-loaded bone demonstrated that mechanical stimuli considerably up-regulate several molecules early after loading, which are known chemoattractants for MSC (Mantila Roosa et al., 2011), including CCL2, C-C motif ligand 7 (CCL7) (Fox et al., 2007; Schenk et al., 2007; Shinohara et al., 2011) and PDGF (Mantila Roosa et al., 2011). This suggests that systemically applied MSC could potentially be recruited and engraft in mechanically induced bone formation, however, to the best of our knowledge, related studies are lacking.

Here, we investigated whether systemically applied MSC are able to support bone formation induced either by an injury or a mechanical stimulus, using a femur osteotomy model and a non-invasive ulna loading model, respectively, in mice. We found that MSC were recruited to the injury site after fracture and increased bone formation. However, in mechanically induced bone formation, we failed to detect injected cells in the newly formed bone, and bone apposition was not enhanced, implying that circulating MSC are not involved in mechanically induced bone formation.

\section{Materials and Methods}

\section{Animal model and husbandry}

All experimental procedures were performed according to the national and international regulations for the care and use of laboratory animals and were approved by the responsible ethics committee (Germany, Regierungspräsidium Tübingen, No. 1029). In total, 76 male C57BL/6J mice were purchased from Charles River (Sulzfeld, Germany). The animals were housed in groups of up to four animals per cage with a $14 \mathrm{~h}$ light, $10 \mathrm{~h}$ dark cycle. Food and water were available ad libitum. EGFP-transgenic mice (strain C57BL/6-Tg(CAG-EGFP)131Osb/LeySop/J) for cell isolation were purchased from Jackson Laboratories (Bar Harbor, USA).

\section{Study design}

To investigate the effect of systemically applied MSC on bone formation during fracture healing and load-induced bone modelling, we used a femur osteotomy model and a non-invasive ulna-loading model, respectively. In the fracture-healing experiment, the mice received a single intravenous injection of either $1 \times 10^{6}$ EGFP-labelled MSC in $100 \mu \mathrm{L}$ phosphate-buffered saline (PBS, PAA Laboratories, Graz, Austria) with 10 IU heparin or heparinonly solution $2 \mathrm{~h}$ after the creation of a femur osteotomy. To investigate load-induced bone formation, the mice received either two injections of $1 \times 10^{6}$ EGFP-labelled MSC or vehicle, respectively, on days 2 and 8 of the loading experiment (described below).

\section{Cell Isolation and Cultivation}

To isolate MCS bone marrow from femora and tibiae from EGFP-transgenic mice (strain C57BL/6-Tg(CAGEGFP)131Osb/LeySop/J), the bones were flushed using PBS (PAA Laboratories). A single cell suspension was achieved using a $70 \mu \mathrm{m}$ cell strainer. After washing with PBS, the cells were seeded at 400,000 to 500,000 cells $/ \mathrm{cm}^{2}$ in growth medium consisting of DMEM/F-12 (1:1) $\left(\mathrm{Gibco}^{\circledR}\right.$, Life Technologies, Darmstadt, Germany), supplemented with $10 \%$ foetal bovine serum MSC Qualified (Gibco ${ }^{\circledR}$, Life Technologies), $1 \%$ L-glutamine (PAA Laboratories), $1 \%$ penicillin-streptomycin and $50 \mu \mathrm{M} 2$-mercaptoethanol (both Gibco ${ }^{\circledR}$, Life Technologies). The cells were cultivated at $37{ }^{\circ} \mathrm{C}$ under $6 \% \mathrm{O}_{2}, 8.5 \% \mathrm{CO}_{2}$ and saturated humidity. After passage zero, the cells were depleted of CD11bpositive cells using an automated magnetic cell sorting system (MACS, Miltenyi, Bergisch-Gladbach, Germany) according to the manufacturer's protocol. Purified MSC were seeded at 1,000-1,500 cells $/ \mathrm{cm}^{2}$ and cultivated under the same conditions as stated above. MSC in passages four to six were used for injection.

Serum in the medium was reduced to $5 \%, 24 \mathrm{~h}$ prior to injection. After two washing steps using PBS, the cells were detached using Accutase $\left(5 \mathrm{~mL} / 175 \mathrm{~cm}^{2}\right.$, PAA Laboratories). The cells were again washed using PBS, resuspended and counted using trypan blue. To prevent clotting of the cells, MSC for injection were resuspended at $10 \times 10^{6}$ cells $/ \mathrm{mL}$ in PBS with $100 \mathrm{IU}$ heparin $/ \mathrm{mL}$ (Heparin-sodium, Ratiopharm, Ulm, Germany) (Deak et al., 2010). An injection of a $100 \mu \mathrm{L}$ cell suspension $\left(1 \mathrm{x} 10^{6}\right.$ MSC) or $100 \mu \mathrm{L}$ PBS with heparin was performed into the lateral tail vein of the mice following the respective regimen.

\section{In-vivo studies}

Fracture healing

To investigate the effect of systemically applied MSC on fracture healing, we created a standardised osteotomy at the femur of 12-week-old mice as described previously in detail by our group (Röntgen et al., 2010). The osteotomy was created under general anaesthesia (2\% Isoflurane, Forene $^{\circledR}$, Abbott, Wiesbaden, Germany) using a Gigli-wire saw $(0.44 \mathrm{~mm})$ and stabilised using an external fixator (RISystem AG, Davos, Switzerland), which was fixed to the cranio-lateral side of the femur using four mini-Schanz screws (Röntgen et al., 2010). The injection of MSC or vehicle solution into the lateral tail vein was performed $2 \mathrm{~h}$ after osteotomy in order to prevent interference of the heparin in the solution with blood coagulation. For pain medication, the mice were treated with $25 \mathrm{mg} / \mathrm{L}$ tramadol 
hydrochloride Tramal $^{\circledR}$, Gruenenthal GmbH, Aachen, Germany) via drinking water 1 day pre-surgery until 3 days post-surgery. To prevent infections, the mice received a single dose of $45 \mathrm{mg} / \mathrm{kg}$ antibiotics subcutaneously (Clindamycin $150 \mathrm{mg} / \mathrm{mL}$, Ratiopharm). The mice were euthanised 3, 10 and 21 days after surgery after blood withdrawal.

\section{Non-invasive ulna loading}

To study the influence of MSC treatment on load-induced bone formation, the right ulnae of 18-week-old mice were subjected to non-invasive mechanical loading (Lee et al., 2002). For loading, the ulna was positioned between two silicon-padded cylinders. Cyclic axial compression of the ulna was performed using a maximum load of $1.5 \mathrm{~N}$ at a frequency of $2 \mathrm{~Hz}$ for $1 \mathrm{~min}$. The loading was applied on five consecutive days per week for 2 weeks. The left ulnae served as internal, non-loaded controls. In order to increase the number of circulating MSC over a prolonged time, the mice received an injection of either $100 \mu \mathrm{L}$ MSC solution (MSC-treatment group) or heparin solution (vehicle group) on day 2 and 8 of the regimen. For dynamic histomorphometry, the mice received an intraperitoneal injection of calcein green $(0.03 \mathrm{~g} / \mathrm{kg})$ on day 3 and of alizarin red S $(0.045 \mathrm{~g} / \mathrm{kg})$ (both Sigma, Steinheim, Germany) on day 12 . The mice were euthanised 16 days after the first loading.

\section{Biomechanical testing}

To determine the mechanical properties of the healed femora, non-destructive three-point bending was performed 21 days post-surgery (Heilmann et al., 2013). For testing, the femoral heads were fixed to aluminium cylinders (Ø $8 \mathrm{~mm}$ ) using a two-component adhesive (i-Cem ${ }^{\circledR}$ SelfAdhesive, Heraeus Kulzer, Hanau, Germany). The cylinder was fixed in a material testing machine (Z10, Zwick Roell, Ulm, Germany) serving as proximal support for the bending test. The condyle of the femur was placed on the distal, unfixed bending support. The bending load, F, was applied to the top of the callus and a force-displacement curve was recorded using a crosshead speed of $2 \mathrm{~mm} / \mathrm{min}$ up to a maximum of $4 \mathrm{~N}$. The flexural rigidity $\mathrm{E}^{*} \mathrm{I}$ was calculated from the linear elastic region of the force-displacement curve (Heilmann et al., 2013; Röntgen et al., 2010).

\section{Micro-computed tomography}

Ulnae and femora harvested after 21 days were scanned using a micro-computed tomography $(\mu \mathrm{CT})$ device (Skyscan 1172, Skyscan, Kontich, Belgium) with a resolution of $8 \mu \mathrm{m}$ per pixel at a peak voltage of $50 \mathrm{kV}$ and $200 \mathrm{~mA}$. Within each scan, phantoms with a defined content of hydroxyapatite - 250 and $750 \mathrm{mg} \mathrm{HA} / \mathrm{cm}^{3}-$ were scanned to determine the bone mineral density.

From reconstructions of the femora, the standard ASBMR parameters (Parfitt, 1987), total tissue volume (TV), bone volume (BV) and bone volume fraction (BV/ TV) were determined by segmentation of the former osteotomy gap using the CT analysis software (CTAnalyser, Skyscan). To distinguish between mineralised and nonmineralised tissue, a global threshold corresponding to a grey-value of $641.9 \mathrm{mg} \mathrm{HA} / \mathrm{cm}^{3}$ was used (Morgan et al., 2009).

Whole ulnae were segmented using the same threshold as above. A $1 \mathrm{~mm}$ thick volume of interest beginning $3.5 \mathrm{~mm}$ distally from the ulna middle was analysed for the parameters BV, moment of inertia (MMIx) and cortical width (Ct.Wi.).

\section{Histomorphometrical analysis}

Bones explanted after 3, 10 and 21 days for histochemical analysis were fixed in $4 \%$ formalin for a minimum of 2 days, decalcified in $20 \%$ ethylenediamine tetraacetic acid, dehydrated and embedded in paraffin (Paraplast ${ }^{\circledR}$ Plus, McCromick Scientific). From the femora, $6 \mu \mathrm{m}$ longitudinal sections were cut using a precision microtome (CUT 6062, SLEE, Mainz, Germany), whereas the ulnae were cut transversally. For histomorphometric analysis, the femora were stained using Safranin-O/Fast Green.

Additional femora, from mice euthanised after 21 days, were embedded in methyl methacrylate (MMA), cut longitudinally with $6 \mu \mathrm{m}$ thickness and stained using Giemsa.

To determine the bone formation rate (BFR) in the ulnae, $10 \mu \mathrm{m}$ thick cross-sections from the MMAembedded bones were cut $4 \mathrm{~mm}$ distally from the ulnamiddle and covered without staining.

Stained sections from the osteomised femora were scanned at 50-fold magnification using a light microscope (Leica DMI6000 B, Leica, Heerbrugg, Switzerland). The amounts of fibrous tissue (FT), cartilage $(\mathrm{Cg})$ and bone (TOT, total osseous tissue) were determined by tracing the circumference of the related tissue area using image analysis software (Leica MMAF 1.4.0 Imaging System, Leica).

Sections from the ulnae were scanned at 200 -fold magnification using a fluorescence microscope (Leica DMI6000 B) with filters for red and green fluorescence. Using image analysis software (Leica MMAF 1.4.0 Imaging System, Leica), the endocortical and periosteal bone surface (BS, circumference), single- and doublelabelled surface and inter-label width were determined. Using these parameters, the endosteal and periosteal BFRs (MARxMS $/ \mathrm{BS} ; \mu \mathrm{m}^{3} / \mu \mathrm{m}^{2} / \mathrm{d}$ ) were calculated (Parfitt, 1987).

\section{Immunohistological staining for EGFP and SDF-1}

For immunohistochemical staining, sections were deparaffinised in xylene and rehydrated. Antigen retrieval was performed by immersion in citric acid buffer $\mathrm{pH} 6$ at $95{ }^{\circ} \mathrm{C}$ for $20 \mathrm{~min}$. For EGFP staining, nonspecific binding sites were blocked using $10 \%$ normal goat serum (Jackson ImmunoResearch Europe Ltd., Newmarket, Suffolk, UK) in tris-buffered saline $\mathrm{pH} 7.6$ with $0.1 \%$ Triton X-100 (Sigma-Aldrich) (TTBS) for $1 \mathrm{~h}$ at room temperature. The sections were incubated overnight at $4{ }^{\circ} \mathrm{C}$ with $2 \mu \mathrm{g} / \mathrm{mL}$ anti-EGFP antibody (rabbit polyclonal, Abcam, Cambridge, UK) or $2 \mu \mathrm{g} / \mathrm{mL}$ isotype control (rabbit immunoglobulin G, Dianova, Hamburg, Germany). Incubation with a biotinylated goat anti-rabbit secondary antibody (1:100, Molecular Probes ${ }^{\circledR}$, Life Technologies, 


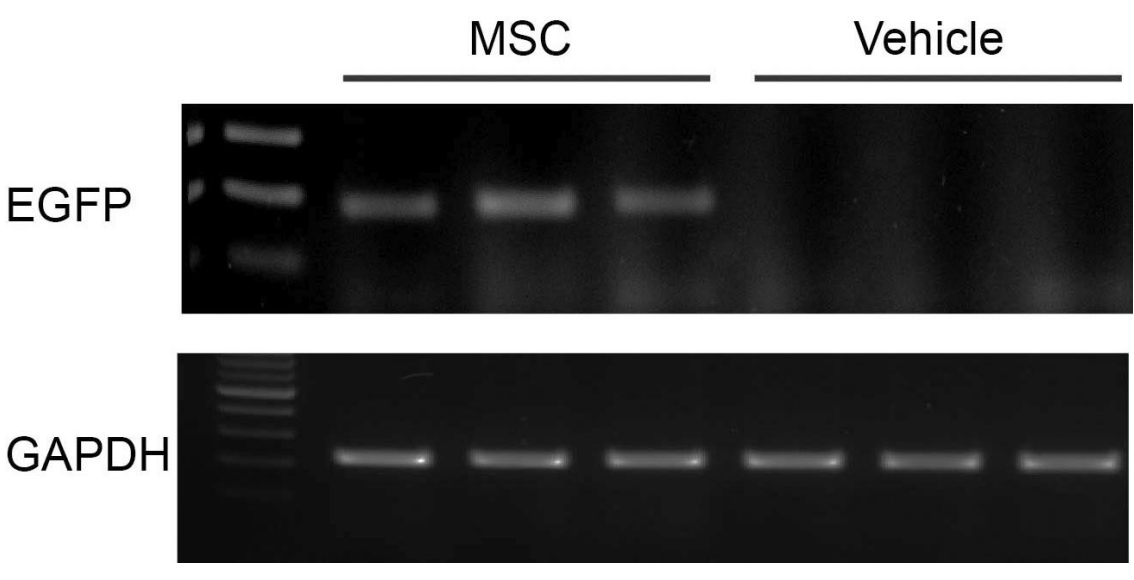

Fig. 1. Representative PCR results of lung lysates of mice after the application of EGFP-transgenic MSC (upper gel, lane 1-3 next to marker) or vehicle (upper gel, lane 4-6). GAPDH served as control for successful PCR (lower gel).
Darmstadt, Germany) was performed at room temperature for $45 \mathrm{~min}$. For signal amplification, HRP-linked streptavidin (Zytomed, Berlin, Germany) was applied on the sections for $15 \mathrm{~min}$. Subsequently, 3-amino-9ethylcarbazole single solution (Zytomed, Berlin, Germany) was used for staining. Staining for alkaline phosphatase was performed as described above. For blocking, $5 \%$ horse serum (Gibco ${ }^{\circledR}$, Life Technologies, Darmstadt, Germany) in TTBS was applied. The sections were incubated at $4{ }^{\circ} \mathrm{C}$ overnight with $2 \mu \mathrm{g} / \mathrm{mL}$ anti alkaline phosphatase antibody (goat anti-mouse, R\&D Systems, Minneapolis, USA) or $2 \mu \mathrm{g} / \mathrm{mL}$ isotype control (goat immunoglobulin G, Sigma-Aldrich, Hamburg. Germany). Incubation with a biotinylated donkey anti-goat antibody (1:100, Santa Cruz Biotechnology, Dallas, USA) was applied at room temperature for $1 \mathrm{~h}$. Signal amplification and detection were performed as described above.

To stain for SDF-1, sections were blocked using $10 \%$ goat serum in TTBS and incubated with $5 \mu \mathrm{g} / \mathrm{mL}$ primary antibody (rabbit polyclonal, Novus Biologicals, Littleton, $\mathrm{CO}$, Canada) or $5 \mu \mathrm{g} / \mathrm{mL}$ isotype control (rabbit immunoglobulin G, Dianova, Seelze, Germany) overnight at $4{ }^{\circ} \mathrm{C}$. The sections were incubated with biotinylated secondary antibody (1:100, Molecular Probes ${ }^{\circledR}$, Life Technologies) for $45 \mathrm{~min}$ at room temperature. For signal detection, Vectastain Elite ABC kit and Vector NovaRED substrate (both Vector laboratories Inc. Burlingame, CA, USA) were used according to the manufacturer's instructions. All sections were counterstained using haematoxylin.

\section{Polymerase chain reaction}

To determine the presence of EGFP-transgenic MSC in tissues other than bone, the lungs, brain, heart, liver, kidneys and spleen of the mice were snap-frozen in liquid nitrogen. To isolate genomic DNA, the organs were ground (MM 400, Retsch, Haan, Germany) and processed according to the manufacturer's protocol using the DNeasy kit (Qiagen, Hilden, Germany). For the polymerase chain reaction (PCR), $1 \mu \mathrm{g}$ DNA was used (HotStarTaq Master Mix, Qiagen; annealing $62{ }^{\circ} \mathrm{C}$ for $45 \mathrm{~s}$, elongation $72{ }^{\circ} \mathrm{C}$ for $30 \mathrm{~s}, 40$ cycles) with the following primers (sequences from Jackson Laboratories): EGFP forward: 5'-AAG TTC ATC TGC ACC ACC G-3'; EGFP reverse: 5'- TCC TTG AAG AAG ATG GTG CG-3' (173 bp); GAPDH forward: 5'-
CCC GTT TGC AAC ATG GCG GC-3'; GAPDH reverse 5'- GCG CCC GTT CAG ACC CAT CC-3' (214 bp). Amplification products were separated on $1.5 \%$ agarose gels and stained using ethidium bromide.

\section{Serum analysis}

Serum obtained from mice treated with MSC or vehicle was analysed 3 days after surgery for IL-1 $\beta$, IL-6, IL-10, tumour necrosis factor (TNF)- $\alpha, \mathrm{MCP}-1$ (CCL2), and macrophage inflammatory protein (MIP)-1 $\alpha$ using the Bio-Plex Pro Cytokine Assay (Bio-Rad, Hercules, CA), according to the manufacturer's instructions. Cytokine concentrations were automatically calculated (Bio-Plex Manager Software 4.1, Bio-Rad), using the standard curve of cytokine standards.

\section{Statistical analysis}

For statistical analysis, SPSS-software (Version 21, IBM Corp., Chicago, IL, USA) was used. Unless stated otherwise, results are depicted as box and whiskers plots. For comparisons between the vehicle and MSC-treated groups, a non-parametric Mann-Whitney-U-test was used. To compare results within a treatment group, a paired Mann-Whitney-U-test was applied. $p \leq 0.05$ was considered to be statistically significant.

\section{Results}

\section{Intravenously injected MSC are recruited into the evolving fracture callus}

To confirm the successful intravenous injection of the EGFP-transgenic MSC and their distribution, DNA isolated from the lung, brain, kidney, heart, liver and spleen at the various time points after MSC treatment was applied to PCR. The EGFP-transgene was detected in the lung at the investigated time points, days 3, 10, 16 and 21 (Fig. 1). Sporadically, the transgene was also detected in the heart, liver and kidneys at days 10 and 16 (data not shown). The positive signal in the lung, as proof for successful injection, was used as an inclusion criterion for further investigations. As expected, no transgene was found in any of the vehicletreated animals.

Immunohistological staining for EGFP in osteotomised femora of the MSC-treated mice showed engraftment of MSC in bone marrow at day 3 and in the evolving callus 
Fig. 2. Immunohistochemical staining for EGFP in longitudinal sections of the fracture calli of mice treated with EGFP-transgenic MSC, 10 days after fracture. (a) Overview of the whole callus, Safranin-O staining: cartilage is stained red-orange and bone is stained light blue; calibration bar $=500 \mu \mathrm{m}$. (b-d) Magnifications of the transition zone of endosteal callus and the bone marrow (b), the cartilaginous partition of the callus (c) and zones of woven bone formation (d). Positively stained cells are found in regions of woven bone. In b-d, calibration bar $=50 \mu \mathrm{m}$.
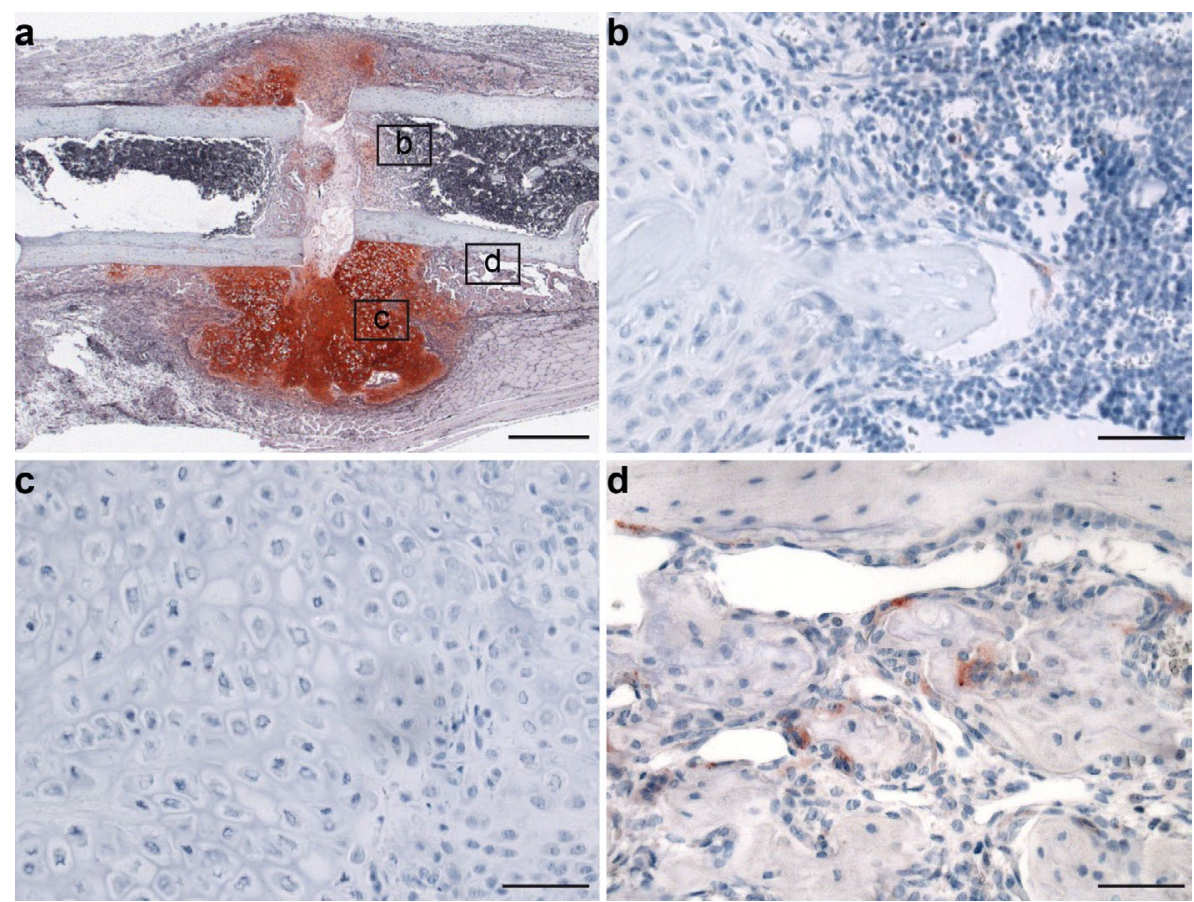

at days 10 and 21. Ten days after fracture, the EGFPlabelled cells were located in the peripheral callus in regions of woven bone formation, but not in the central, cartilaginous fraction of the callus (Fig. 2). These cells appeared to adopt a cuboidal, osteoblast-like shape. At day 21, EGFP-labelled cells were located at the margins of newly formed bone trabeculae (Fig. 3, right column), again having an osteoblast-like phenotype. No EGFPlabelled cells were detected in the cartilaginous regions of the callus, but were present in the transition zone of the cartilage and woven bone. As expected, no EGFPcells were detected in vehicle-treated animals (Fig. 3, left column). Furthermore, no positive signals were found in the intact femora of osteotomised or uninjured mice (data not shown). Comparing day 10 and 21, an increase in the number of EGFP-positive cells was evident ( $16 \pm 3$ cells/ $\mathrm{mm}^{2}$ on day 10 and $152 \pm 56$ cells $/ \mathrm{mm}^{2}$ callus area on day 21). Staining of adjacent sections for EGFP and alkaline phosphatase implies participation of the injected cells at the process of bone formation as similar regions were stained (Fig. $3 \mathrm{i}$ and j).

Staining for SDF-1 (CXCL-12), a chemokine that is known to be involved in the key mechanism for stem cell recruitment (Ceradini et al., 2004; Otsuru et al., 2008), showed positively stained cells in the osteotomy region (Fig. $4 \mathrm{a}$ and b) and the stroma (Fig. $4 \mathrm{c}$ and d) of the osteotomised femora 3 days after surgery. In the stroma, virtually all the cells were positively stained. There were no obvious differences between MSC-and vehicle-treated animals.

\section{Bone formation in fracture healing is increased by intravenous injection of MSC}

$\mu \mathrm{CT}$ analysis of the former osteotomy gap in the right femora $21 \mathrm{~d}$ after surgery demonstrated a significant increase in BV by $49 \%$ in the MSC-treated group compared to the vehicle group ( $p=0.026$, Fig. 5 b). TV and $\mathrm{BV} / \mathrm{TV}$ were also slightly increased in the MSC group by $21 \%$ and $24 \%$, respectively; however, these differences did not reach statistical significance (Fig. 5 a and $\mathrm{c}$ ). The bending stiffness of the fracture calli was not significantly improved by the MSC treatment (Fig. $5 \mathrm{~d})$. Histomorphometric analysis of the fracture calli at day 10 revealed no statistical significant differences (Fig. $5 \mathrm{e}$ ); however analysis of the callus composition on day 21 demonstrated an increase in newly formed bone $(+60 \%)$, confirming the $\mu \mathrm{CT}$ analysis (Fig. $5 \mathrm{f}$ ); however this increase did also not reach statistical significance.

\section{Pro- and anti-inflammatory cytokine levels are not altered by systemic MSC application}

Multiplex analysis demonstrated no significant differences in the serum levels of IL-1 $\beta$, IL-6, IL-10, TNF $\alpha$ and MCP1 three days after osteotomy in mice treated with MSC or vehicle solution. The values obtained were largely in the physiological range (Knoferl et al., 2003), while only $\mathrm{TNF} \alpha$ appeared to be slightly elevated.

\section{Systemically administered MSC do not engraft at sites of mechanically induced bone formation}

Immunohistochemical staining for EGFP in ulnae showed no engraftment of MSC in non-loaded (not shown) or loaded ulnae in the MSC-treated group in the endosteal or periosteal regions (Fig. $6 \mathrm{~b}$ and d). SDF-1 expression was detected in the stroma of loaded (Fig. 6 e and f) and non-loaded (not shown) ulnae with no obvious differences. Osteocytes in the cortex were negative for SDF-1 (Fig. 6 $\mathrm{g}$ and $\mathrm{h}$ ). In vehicle-treated mice, no EGFP-positive cells were detected (Fig. 6 a and c). 

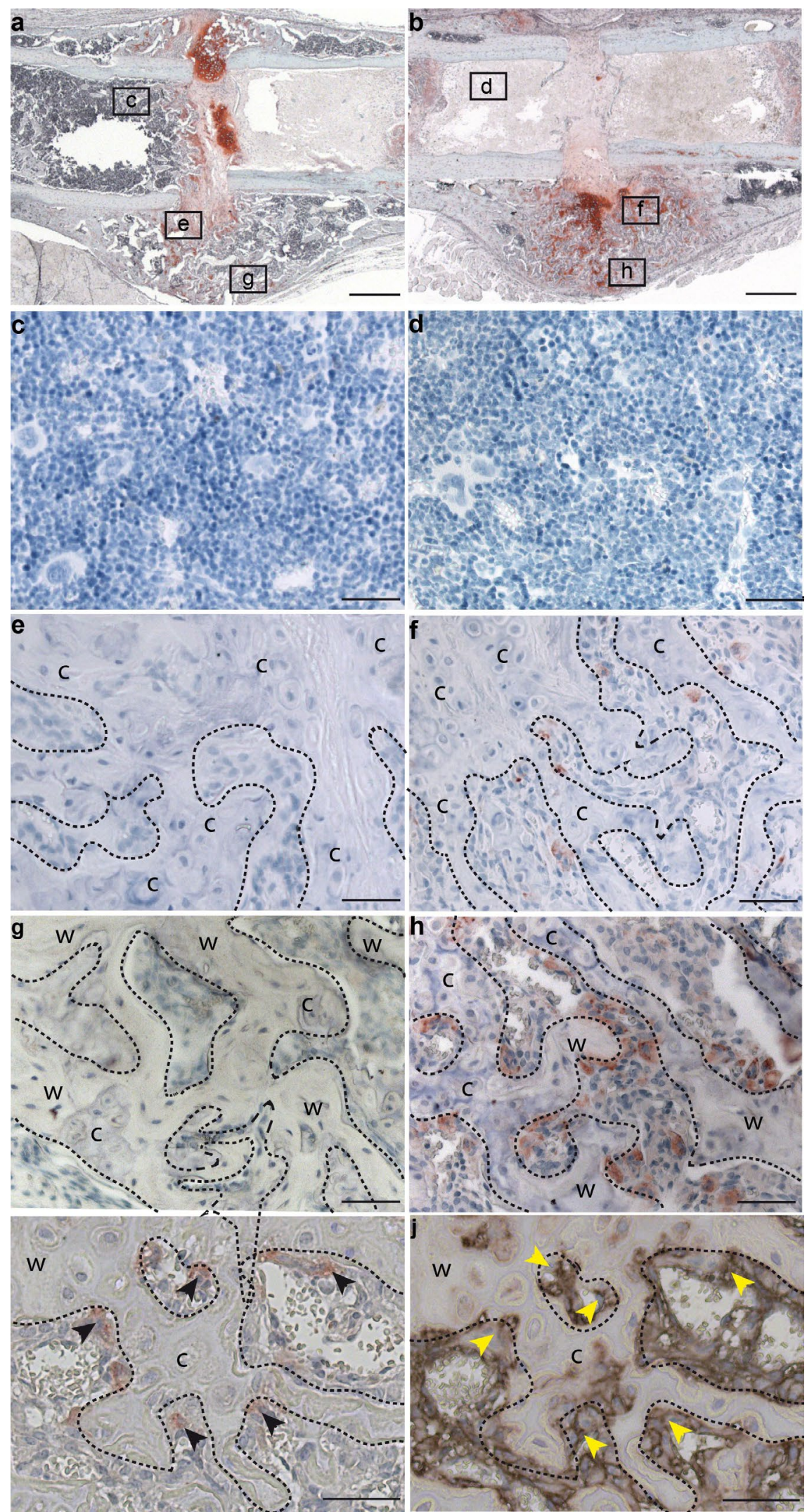

Fig. 3. Immunohistochemical staining for EGFP in longitudinal sections of fracture calli of vehicle $(\mathbf{a}, \mathbf{c}, \mathbf{e}$ and $\mathbf{g})$ and EGFP-transgenic MSC-treated (b, d, $\mathbf{f}$ and $\mathbf{h}$ ) mice 21 days after fracture. (a and $\mathbf{b}$ ): Overview over the whole callus of the vehicle (a) and MSC-treated mice (b). Safranin-O staining: cartilage is stained red-orange and bone is stained light-blue; calibration bar $=500 \mu \mathrm{m}$. (c-h): Magnifications of the bone marrow $(\mathbf{c}, \mathbf{d})$, the cartilaginous partition of the callus $(\mathbf{e}, \mathbf{f})$ and zones of woven bone $(\mathbf{g}, \mathbf{h})$. Positively stained cells are present at the margin of bone trabeculae in MSC-treated mice (f, $\mathbf{h})$. No positively stained cells were detected in the bone marrow or cartilage after MSC treatment (e, f upper right corner). In vehicle-treated mice, no stained cells were detected. Staining for EGFP (i) and alkaline phosphatase (j) in adjacent sections revealed positive staining in similar regions (arrowheads in both figures). In $\mathbf{c}-\mathbf{j}$, calibration bar $=50 \mu \mathrm{m}$. In $\mathbf{e}-\mathbf{j}$, dashed lines indicate the margin of cartilaginous structures (c) or woven bone (w). 
Fig. 4. Staining for SDF-1 in animals treated with vehicle (a, c) and EGFP-transgenic MSC (b, d), 3 days after surgery. In both groups in the osteotomy region (a, b) and the bone marrow (c, d), positive signals for SDF1 were observed. Calibration bar $=50 \mu \mathrm{m}$.
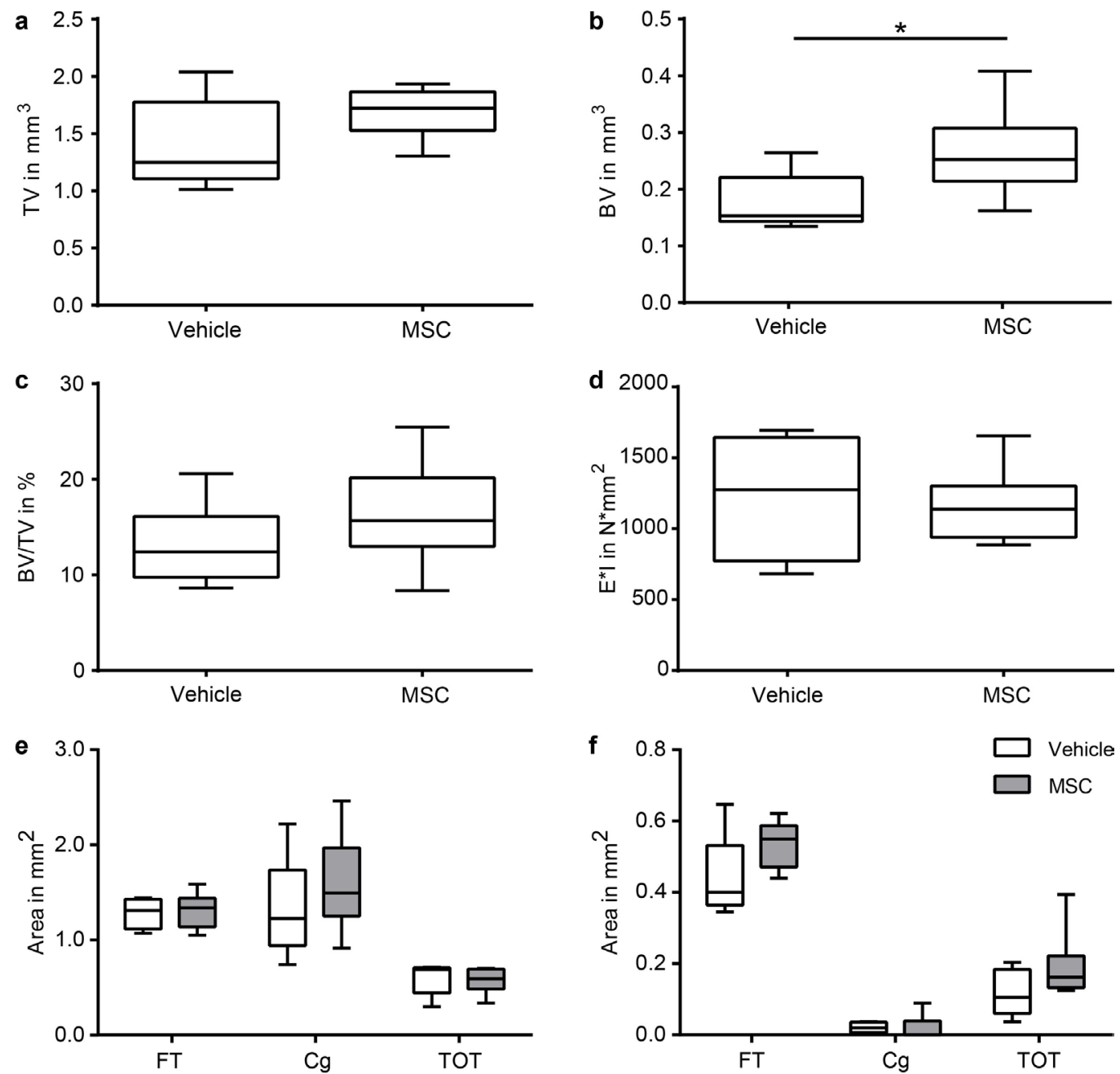

Fig. 5. Analysis of fracture healing using $\mu \mathrm{CT}$ (a-c) and biomechanical testing (d) after 21 days of healing. $\mu \mathrm{CT}$ analysis shows no significant differences in the callus volume (a), however, the bone volume is significantly increased in the MSC treatment group (b). The relative proportion of bone is also increased in the MSC-treatment group, but not significantly (c). Biomechanical testing of the fracture calli shows no differences (d). Histomorphometric analysis of the callus composition on day 10 (e) and 21 (f) revealed no statistical significant differences; however on day 21 an increase in bone content was evident after MSC-treatment. Results are presented as box plots; $n=6$ per group. Asterisk denotes $p<0.05$. 


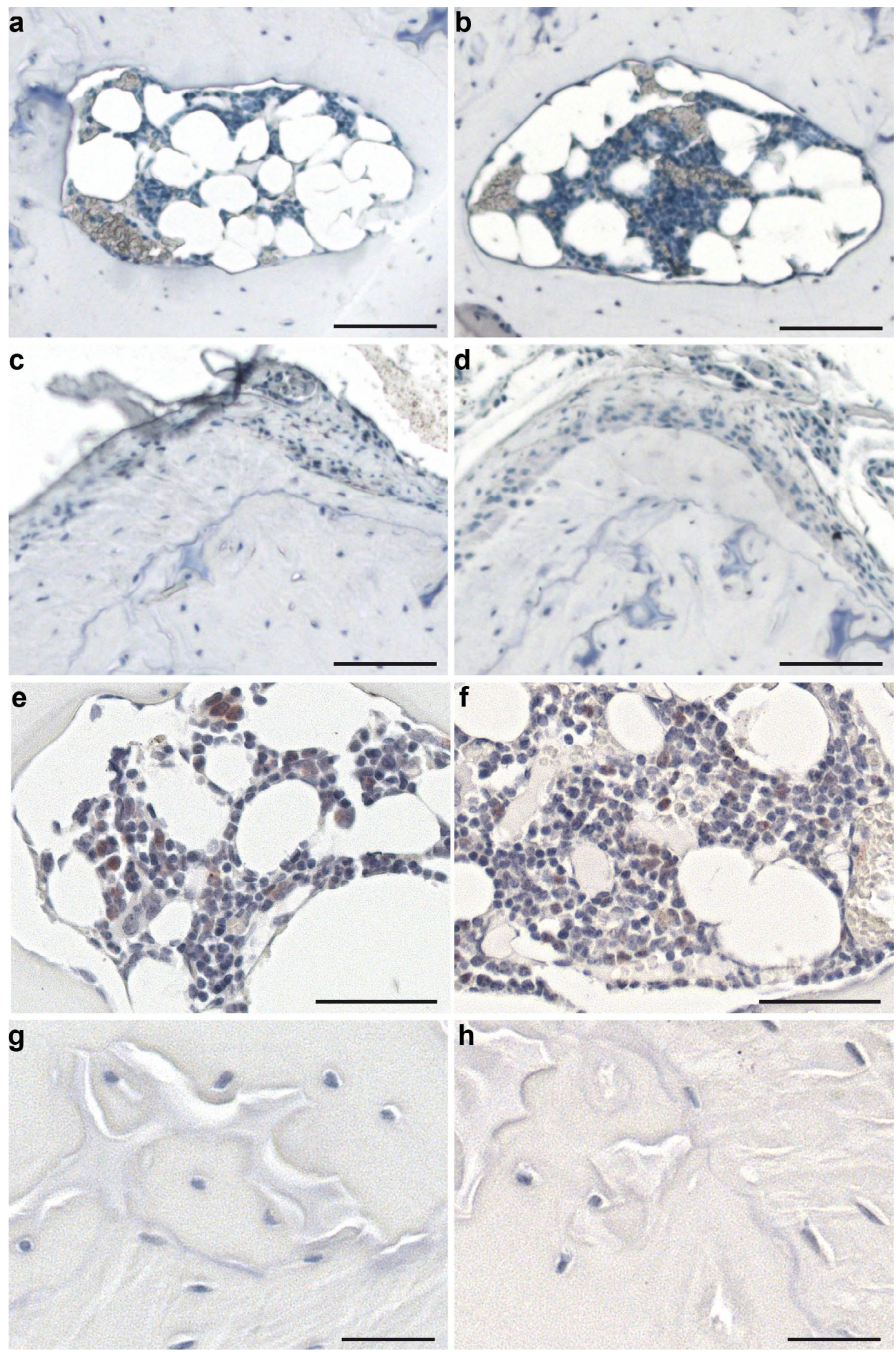

Fig. 6. Immunohistochemical staining for EGFP in endosteal $(\mathbf{a}, \mathbf{b})$ and periosteal $(\mathbf{c}, \mathbf{d})$ regions of loaded ulnae of mice treated with vehicle $(\mathbf{a}, \mathbf{c})$ or EGFP-transgenic MSC $(\mathbf{b}, \mathbf{d})$. No positively stained cells were detected. Calibration bar in $\mathbf{a}-\mathbf{d}=100 \mu \mathrm{m}$. Immunohistochemical staining for SDF-1 in the stroma $(\mathbf{e}, \mathbf{f})$ and cortex $(\mathbf{g}, \mathbf{h})$ of loaded ulnae in mice treated with vehicle $(\mathbf{e}, \mathbf{g})$ or EGFP-transgenic MSC (f, h). Calibration bar in $\mathbf{e}-\mathbf{h}=50 \mu \mathrm{m}$. 
a

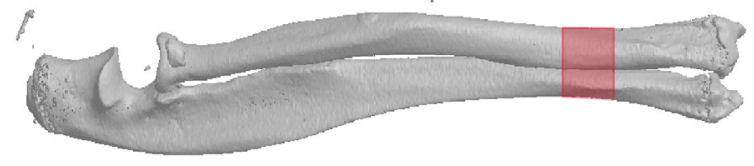

C

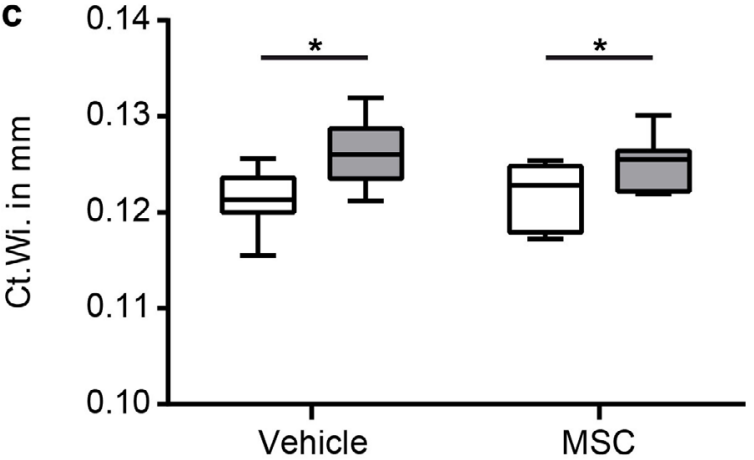

b
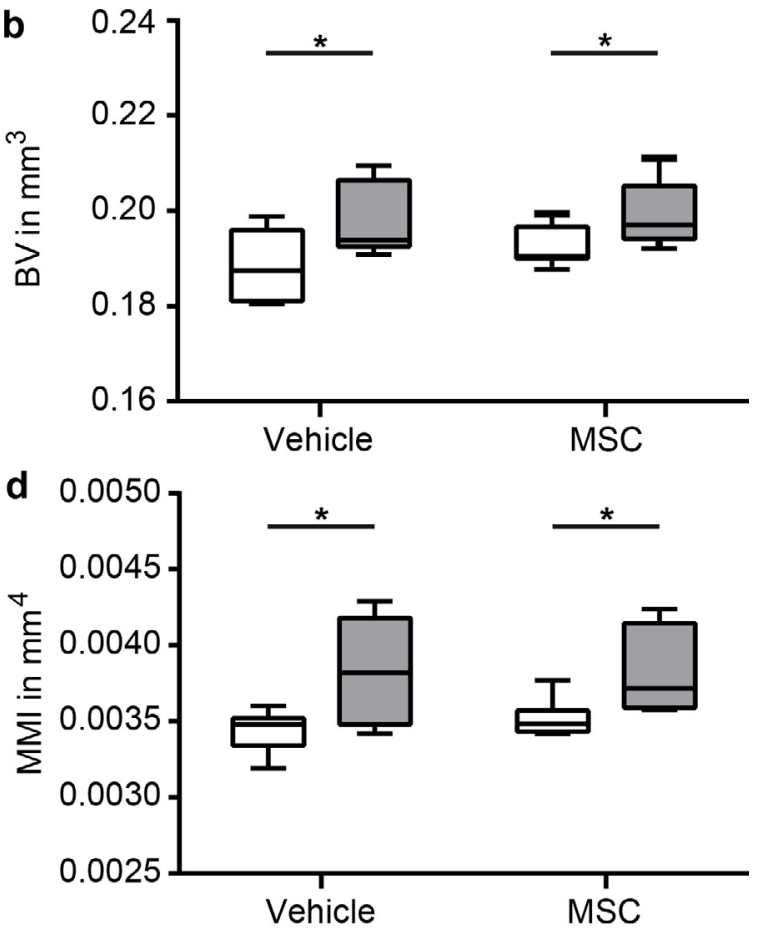

Fig. 7. $\mu \mathrm{CT}$ analysis of bone formation in ulnae after mechanical loading. The region of interest assessed is highlighted in red in a 3D-reconstruction of a right ulna (a). After loading, the bone volume (b), cortical width (c) and moment of inertia (d) increased significantly in loaded (grey bars) relative to non-loaded ulnae (white bars). Comparison of vehicle- and MS-treated mice showed no differences. Results are presented as box plots; $n=7$ per group. Asterisk denotes $p<0.05$.
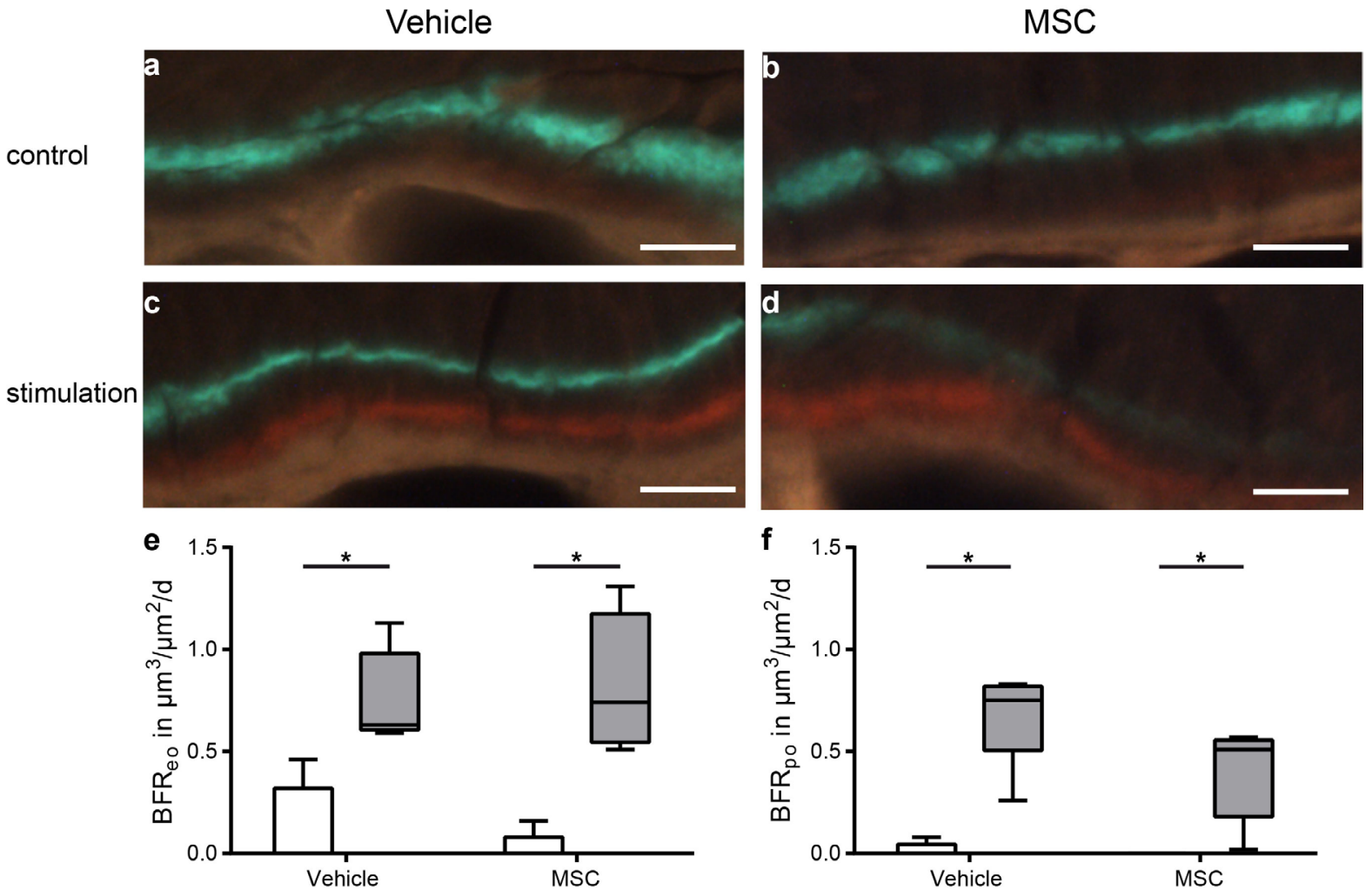

Fig. 8. Analysis of bone formation after mechanical loading in vehicle- and MSC-treated mice using dynamic histomorphometry. For the assessment of dynamic histomorphometrical parameters, calcein green and alizarin red $\mathrm{S}$ were administered on days 3 and 12, respectively. Representative images of the fluorescent endosteal labels are shown in a-d. Calibration bar $=50 \mu \mathrm{m}$. Loading of the bone led to a significant increase in the bone formation rate (BFR) relative to the non-loaded control (grey bar $v s$. white bar) in the endosteal (e) and periosteal (f) locations. No significant differences were detected between vehicle- and MS-treated mice. Results are presented as box plots; $n=5$ per group. Asterisk denotes $p<0.05$. 


\section{Bone formation in mechanically induced bone modelling is not increased by MSC treatment}

$\mu \mathrm{CT}$ analysis of loaded and non-loaded ulnae demonstrated a significant increase in BV, Ct.Wi. and MMIx after loading compared to the unloaded control (Fig. 7 b-d). A comparison between the loaded ulnae of MSC- and vehicletreated mice showed no statistically significant differences. The assessment of dynamic histomorphometric parameters demonstrated a significantly higher BFR in loaded ulnae in both treatment groups (Figure 8 e and f). Again, no differences were detected in the comparison of the BFRs in loaded ulnae from MSC- and vehicle-treated mice.

\section{Discussion}

The aim of our study was to investigate whether systemically administered MSC support bone formation induced either by bone injury or non-invasive mechanical loading. We found the recruitment of the systemically applied cells to the fracture. Furthermore, we detected a positive effect of the injected cells on fracture-induced bone formation, as demonstrated by a significantly increased BV. In contrast, systemically applied MSC did not engraft in ulnae after mechanical loading, and loadinduced bone formation was not enhanced.

Several studies reported the recruitment of intravenously injected osteoprogenitor cells to bone healing sites (Devine et al., 2002; Granero-Molto et al., 2009; Lee et al., 2009b). These studies also demonstrated the participation of the injected MSC in fracture healing. However, the reported effects were only moderate, ranging from no beneficial effect (Devine et al., 2002) to enhanced bone formation with (Granero-Molto et al., 2009) or without (Lee et al., 2009 b) improvement in the mechanical competence of the healed femur. In our study, we confirmed the recruitment of systemically administered MSC to the fracture healing site using immuno-localisation of EGFP-labelled MSC, because we found positively stained cells in the fracture callus during early and later healing periods. Mandatory for progenitor-cell recruitment is a migratory stimulus. We demonstrated that the hypoxia-regulated SDF-1 (CXCL12), a chemokine that is recognised as a key molecule for stem cell attraction, is expressed after osteotomy by virtually all cells - both, in the bone marrow of the osteotomised femur and in the vicinity of the osteotomy. Others have previously shown that CXCR4-SDF1 signalling is important for normal fracture healing and blockade of CXCR4-SDF1 signalling considerably influences fracture healing (Kitaori et al., 2009; Toupadakis et al., 2012). As described in the literature (Devine et $a l ., 2002)$, the recruitment process appears to be timedependent, because more cells were detected in the callus on day 21 compared to day 10 after injection. We also confirmed the often-described presence of the administered cells in the lung, using PCR. Whereas, in contrast to studies investigating MSC-trafficking after intravenous administration (Allers et al., 2004), we were unable to consistently find cells in other tissues. It is hypothesised, that intravenously injected cells are initially retained in the lung and re-enter the circulation in a secondary step
(Fischer et al., 2009; Gao et al., 2001). This so-called first pass effect is the major drawback that has to be overcome in therapeutic approaches aiming to employ intravenous cell injection (Fischer et al., 2009). Proliferation on site might also contribute to increasing cell numbers over time. However, we're not able to distinguish between a first pass effect and proliferation on site. A possibility to investigate this aspect could be the administration of cells expressing different reporters in different stages of the cell cycle.

In accordance with the literature, immunohistological staining for the reporter gene EFGP in sections of fractured femora showed that the injected MSC were located at the seam of newly formed bone. This finding is corroborated by Devine et al. (Devine et al., 2002). The presence of these cells at the seam of newly formed bone, and positive staining for EGFP and alkaline phosphatase in similar regions of adjacent sections, suggests their involvement in the bone formation process. However, an indirect action of the injected MSC on regenerative processes is also plausible. There are reports of beneficial effects of systemically injected human MSC on corneal regeneration, without significant donor cell engraftment (Oh et al., 2012; Roddy et al., 2011). In another study, it is hypothesised that injected MSC that are entrapped in the lung as emboli secrete the anti-inflammatory protein tumour necrosis factor alpha induced protine-6 (TSG-6) (Lee et al., 2009a). A role for indirect action of MSC on regenerative processes is further strengthened by the observation that knock-down of TSG-6 is sufficient to abolish the beneficial effect of MSC-injection in a variety of models (Danchuk et al., 2011; Lee et al., 2009a; Qi et al., 2013).

Our results showing increased bone formation after MSC-injection are also in agreement with the literature, where the formation of a larger callus with significantly greater amounts of newly formed bone was reported (Granero-Molto et al., 2009). Despite the significant increase in the BV in the MSC-treated mice relative to vehicle-treated mice, the callus stiffness and its material properties were not significantly altered, indicating a moderate anabolic effect on bone healing without any functional improvement in our model.

The systemic immunomodulatory action of the injected MSC, that was reported by others (Granero-Molto et al., 2009) even 3 days after fracture, was not evident in our study (data not shown). We did not expect to detect an alteration in the serum cytokine levels, because our previous and on-going studies show that an isolated femur osteotomy is unable to induce any considerable systemic inflammatory response even $6 \mathrm{~h}$ after osteotomy and that the initial post-traumatic systemic inflammatory response is down-regulated to physiological levels within $24 \mathrm{~h}$ of osteotomy in rodents. To achieve a considerable systemic inflammation, a second trauma is necessary (Recknagel et al., 2011). However, whether the applied MSC have an immunomodulatory effect cannot be fully answered by the present study, earlier time-points after fracture would be necessary to investigate this.

The effects applied MSC on bone regeneration are moderate compared to other osteoanabolic treatments, such as administration of bone morphogenetic proteins or parathyroid hormone (Chalidis et al., 2007; Giannoudis 
and Dinopoulos, 2010). At least, a singular systemic administration of MSC seems ineffective in significantly improving fracture healing. Hence, a local application of stem cells seems a better approach for treatment of poorly healing fractures in clinics. It is worth mentioning that a systemic transplantation of unmanipulated bone marrow, containing MSC, was effective in treatment of severe Osteogenesis imperfecta. The treatment resulted in marked increases in longitudinal growth and bone mineralisation, indicating the osteogenic potential of systemically applied cells (Horwitz et al., 1999; Horwitz et al., 2001). However, these data are difficult to relate to the findings of the present study, as the mice used for our study received an isolated fracture and did not suffer from a generalised bone disorder.

Generally, the effect of cells transplanted locally into bone defects appears to be stronger than that of systemically delivered cells. Local delivery of cells leads to an immediate increase in the progenitor cell numbers to a super-physiological level and thus more pronounced cell-based effects are likely. In contrast, a considerable increase in the progenitor cell number at the fracture site after systemic injection is unlikely, because cell recruitment remains at a physiological level. Furthermore, a limitation of our study and those of other authors may be that the effect of systemic MSC treatment was investigated in young, healthy mice with good regenerative capacity.

To our knowledge, this study is the first to investigate the role of systemically applied MSC in load-induced bone formation. Due to the expectation, that circulating osteoblast precursors could be recruited into bone remodelling compartments in physiological bone remodelling (Hauge et al., 2001) and because several chemokines are up-regulated after mechanical loading (Mantila Roosa et al., 2011), we hypothesised that systemically administered MSC are recruited in load-induced bone modelling. However, we were unable to detect engraftment of the administered cells at sites of load-induced bone formation. We did not detect regulation of SDF-1 by mechanical loading; however, it is possible that other chemokines were regulated. We focused on SDF-1 as it is - as mentioned earlier - supposed to be the key molecule in MSC recruitment. We demonstrated an increase in the BFR in loaded ulnae, as was expected from the literature (Lee et al., 2002). However, we were unable to detect a further enhancement of bone formation after systemic MSC delivery. Apparently, the local stimulus and the subsequent up-regulation of several chemokines, including CCL2, CCL7 and PDGF, as described in the literature (Mantila Roosa et al., 2011), is insufficient to attract the injected cells to the bone-formation site. Therefore, the increased bone formation is most likely to result from an activation of formerly quiescent bone-lining cells that were activated by mechanical stimuli (Pead et al., 1988) or by the recruitment of local or perivascular MSC or progenitor cells.

Our results suggest the necessity of an adequate stimulus for a stem-cell-based regenerative action. This is because we demonstrated a positive effect of systemically delivered MSC in bone healing, where a more pronounced local and systemic inflammatory environment was evident; but not in load-induced bone formation, where presumably only local alterations in chemokine signalling occur.
Studies showed a strong chemotaxis of MSC towards SDF1 , a chemokine that is regulated by hypoxia, thus being up regulated in fractures (Kitaori et al., 2009).

In conclusion, we demonstrated a beneficial effect of intravenously delivered MSC in fracture healing, shown by a significant increase in bone volume in the MSC-treated group relative to vehicle treatment. However, we found no benefit to load-induced bone formation. Questions that remain to be answered include, whether the beneficial effect on fracture healing is a direct effect of the cells themselves or whether the cells secrete trophic factors that foster the potential of resident cells. Furthermore, the question has to be addressed whether the homing potential of MSC is solely dependent on CXCR4 expression, or whether other molecules are able to recruit MSC in a similar way.

\section{Acknowledgements}

The authors acknowledge the excellent technical assistance of Daniela Kuschel, Sevil Essig, Marion Tomo, Ursula Maile and Helga Bach.

\section{References}

Allers C, Sierralta WD, Neubauer S, Rivera F, Minguell JJ, Conget PA (2004) Dynamic of distribution of human bone marrow-derived mesenchymal stem cells after transplantation into adult unconditioned mice. Transplantation 78: 503-508.

Atesok K, Li R, Stewart DJ, Schemitsch EH (2010) Endothelial progenitor cells promote fracture healing in a segmental bone defect model. J Orthop Res 28: 1007-1014.

Caplan AI (2007) Adult mesenchymal stem cells for tissue engineering versus regenerative medicine. J Cell Physiol 213: 341-347.

Ceradini DJ, Kulkarni AR, Callaghan MJ, Tepper OM, Bastidas N, Kleinman ME, Capla JM, Galiano RD, Levine JP, Gurtner GC (2004) Progenitor cell trafficking is regulated by hypoxic gradients through HIF-1 induction of SDF-1. Nat Med 10: 858-864.

Chalidis B, Tzioupis C, Tsiridis E, Giannoudis PV (2007) Enhancement of fracture healing with parathyroid hormone: preclinical studies and potential clinical applications. Expert Opin Investig Drugs 16: 441-449.

Chandrasekhar KS, Zhou H, Zeng P, Alge D, Li W, Finney BA, Yoder MC, Li J (2011) Blood vessel wallderived endothelial colony-forming cells enhance fracture repair and bone regeneration. Calcif Tissue Int 89: 347-357.

Claes L, Recknagel S, Ignatius A (2012) Fracture healing under healthy and inflammatory conditions. Nat Rev Rheumatol 8: 133-143.

Colnot C, Zhang X, Knothe Tate ML (2012) Current insights on the regenerative potential of the periosteum: Molecular, cellular, and endogenous engineering approaches. J Orthop Res 30: 1869-1878.

Danchuk S, Ylostalo JH, Hossain F, Sorge R, Ramsey A, Bonvillain RW, Lasky JA, Bunnell BA, Welsh DA, Prockop DJ, Sullivan DE (2011) Human multipotent stromal cells attenuate lipopolysaccharide-induced acute 
lung injury in mice via secretion of tumor necrosis factoralpha-induced protein 6. Stem Cell Res Ther 2: 27.

Deak E, Ruster B, Keller L, Eckert K, Fichtner I, Seifried E, Henschler R (2010) Suspension medium influences interaction of mesenchymal stromal cells with endothelium and pulmonary toxicity after transplantation in mice. Cytotherapy 12: 260-264.

Devine MJ, Mierisch CM, Jang E, Anderson PC, Balian G (2002) Transplanted bone marrow cells localize to fracture callus in a mouse model. J Orthop Res 20: 12321239.

Fiedler J, Etzel N, Brenner RE (2004) To go or not to go: Migration of human mesenchymal progenitor cells stimulated by isoforms of PDGF. J Cell Biochem 93: 990998.

Fischer UM, Harting MT, Jimenez F, Monzon-Posadas WO, Xue HS, Savitz SI, Laine GA, Cox CS (2009) Pulmonary Passage is a Major Obstacle for Intravenous Stem Cell Delivery: The Pulmonary First-Pass Effect. Stem Cells Dev 18: 683-691.

Fox JM, Chamberlain G, Ashton BA, Middleton J (2007) Recent advances into the understanding of mesenchymal stem cell trafficking. Brit J Haematol 137: 491-502.

Gao J, Dennis JE, Muzic RF, Lundberg M, Caplan AI (2001) The Dynamic in vivo Distribution of Bone MarrowDerived Mesenchymal Stem Cells after Infusion. Cells Tissues Organs 169: 12-20.

Giannoudis PV, Dinopoulos HT (2010) BMPs: Options, indications, and effectiveness. J Orthop Trauma 24 Suppl 1: S9-16.

Granero-Molto F, Weis JA, Miga MI, Landis B, Myers TJ, O'Rear L, Longobardi L, Jansen ED, Mortlock DP, Spagnoli A (2009) Regenerative Effects of Transplanted Mesenchymal Stem Cells in Fracture Healing. Stem Cells 27: 1887-1898.

Gusi N, Raimundo A, Leal A (2006) Low-frequency vibratory exercise reduces the risk of bone fracture more than walking: a randomized controlled trial. BMC Musculoskelet Disord 7: 92.

Hauge EM, Qvesel D, Eriksen EF, Mosekilde L, Melsen F (2001) Cancellous bone remodeling occurs in specialized compartments lined by cells expressing osteoblastic markers. J Bone Miner Res 16: 1575-1582.

Heilmann A, Schinke T, Bindl R, Wehner T, Rapp A, Haffner-Luntzer M, Liedert A, Amling M, Ignatius A (2013) Systemic treatment with the sphingosine-1phosphate analog FTY720 does not improve fracture healing in mice. J Orthop Res 31: 1845-1850.

Hernigou P, Poignard A, Beaujean F, Rouard H (2005) Percutaneous Autologous Bone-Marrow Grafting for Nonunions. Influence of the Number and Concentration of Progenitor Cells. J Bone Joint Surg Am 87: 1430-1437.

Horwitz EM, Prockop DJ, Fitzpatrick LA, Koo WWK, Gordon PL, Neel M, Sussman M, Orchard P, Marx JC, Pyeritz RE, Brenner MK (1999) Transplantability and therapeutic effects of bone marrow-derived mesenchymal cells in children with osteogenesis imperfecta. Nature medicine 5: 309-313.

Horwitz EM, Prockop DJ, Gordon PL, Koo WWK, Fitzpatrick LA, Neel MD, McCarville ME, Orchard PJ,
Pyeritz RE, Brenner MK (2001) Clinical responses to bone marrow transplantation in children with severe osteogenesis imperfecta. Blood 97: 1227-1231.

Kitaori T, Ito H, Schwarz EM, Tsutsumi R, Yoshitomi H, Oishi S, Nakano M, Fujii N, Nagasawa T, Nakamura T (2009) Stromal cell-derived factor 1/CXCR4 signaling is critical for the recruitment of mesenchymal stem cells to the fracture site during skeletal repair in a mouse model. Arthritis \& Rheumatism 60: 813-823.

Knoferl MW, Liener UC, Seitz DH, Perl M, Bruckner UB, Kinzl L, Gebhard F (2003) Cardiopulmonary, histological, and inflammatory alterations after lung contusion in a novel mouse model of blunt chest trauma. Shock 19: 519-525.

Kumagai K, Vasanji A, Drazba JA, Butler RS, Muschler GF (2008) Circulating cells with osteogenic potential are physiologically mobilized into the fracture healing site in the parabiotic mice model. J Orthop Res 26: 165-175.

Lee KCL, Maxwell A, Lanyon LE (2002) Validation of a Technique for Studying Functional Adaptation of the Mouse Ulna in Response to Mechanical Loading. Bone 31: 407-412.

Lee RH, Pulin AA, Seo MJ, Kota DJ, Ylostalo J, Larson BL, Semprun-Prieto L, Delafontaine P, Prockop DJ (2009a) Intravenous hMSCs improve myocardial infarction in mice because cells embolized in lung are activated to secrete the anti-inflammatory protein TSG-6. Cell Stem Cell 5: 54-63.

Lee S-W, Padmanabhan P, Ray P, Gambhir SS, Doyle T, Contag C, Goodman SB, Biswal S (2009b) Stem cellmediated accelerated bone healing observed with in vivo molecular and small animal imaging technologies in a model of skeletal injury. J Orthop Res 27: 295-302.

Mantila Roosa SM, Liu Y, Turner CH (2011) Gene expression patterns in bone following mechanical loading. J Bone Miner Res 26: 100-112.

Morgan EF, Mason ZD, Chien KB, Pfeiffer AJ, Barnes GL, Einhorn TA, Gerstenfeld LC (2009) Micro-computed tomography assessment of fracture healing: relationships among callus structure, composition, and mechanical function. Bone 44: 335-344.

Oh JY, Lee RH, Yu JM, Ko JH, Lee HJ, Ko AY, Roddy GW, Prockop DJ (2012) Intravenous mesenchymal stem cells prevented rejection of allogeneic corneal transplants by aborting the early inflammatory response. Mol Ther 20: 2143-2152.

Otsuru S, Tamai K, Yamazaki T, Yoshikawa H, Kaneda Y (2008) Circulating bone marrow-derived osteoblast progenitor cells are recruited to the bone-forming site by the CXCR4/stromal cell-derived factor-1 pathway. Stem Cells 26: 223-234.

Parfitt (1987) Bone Histomorphometry. Journal of Bone and Mineral Research 2: 595-610.

Pead MJ, Skerry TM, Lanyon LE (1988) Direct transformation from quiescence to bone formation in the adult periosteum following a single brief period of bone loading. J Bone Miner Res 3: 647-656.

Qi Y, Jiang D, Sindrilaru A, Stegemann A, Schatz S, Treiber N, Rojewski M, Schrezenmeier H, Vander Beken S, Wlaschek M, Bohm M, Seitz A, Scholz N, Durselen L, Brinckmann J, Ignatius A, Scharffetter-Kochanek K (2013) TSG-6 Released from Intradermally Injected Mesenchymal 
Stem Cells Accelerates Wound Healing and Reduces Tissue Fibrosis in Murine Full-Thickness Skin Wounds. J Invest Dermatol 134: 526-537.

Recknagel S, Bindl R, Kurz J, Wehner T, Ehrnthaller C, Knoferl MW, Gebhard F, Huber-Lang M, Claes L, Ignatius A(2011) Experimental blunt chest trauma impairs fracture healing in rats. J Orthop Res 29: 734-739.

Roddy GW, Oh JY, Lee RH, Bartosh TJ, Ylostalo J, Coble K, Rosa RH, Jr., Prockop DJ (2011) Action at a distance: systemically administered adult stem/progenitor cells (MSCs) reduce inflammatory damage to the cornea without engraftment and primarily by secretion of TNFalpha stimulated gene/protein 6. Stem Cells 29: 1572-1579.

Röntgen V, Blakytny R, Matthys R, Landauer M, Wehner T, Göckelmann M, Jermendy P, Amling M, Schinke T, Claes L, Ignatius A (2010) Fracture healing in mice under controlled rigid and flexible conditions using an adjustable external fixator. J Orthop Res 28: 1456-1462.

Schenk S, Mal N, Finan A, Zhang M, Kiedrowski M, Popovic Z, McCarthy PM, Penn MS (2007) Monocyte chemotactic protein-3 is a myocardial mesenchymal stem cell homing factor. Stem Cells 25: 245-251.

Shinohara K, Greenfield S, Pan H, Vasanji A, Kumagai K, Midura RJ, Kiedrowski M, Penn MS, Muschler GF (2011) Stromal cell-derived factor-1 and monocyte chemotactic protein-3 improve recruitment of osteogenic cells into sites of musculoskeletal repair. J Orthop Res 29: 1064-1069.

Shoji T, Ii M, Mifune Y, Matsumoto T, Kawamoto A, Kwon SM, Kuroda T, Kuroda R, Kurosaka M, Asahara $\mathrm{T}$ (2010) Local transplantation of human multipotent adipose-derived stem cells accelerates fracture healing via enhanced osteogenesis and angiogenesis. Lab Invest 90: 637-649.

Taguchi K, Ogawa R, Migita M, Hanawa H, Ito H, Orimo H (2005) The role of bone marrow-derived cells in bone fracture repair in a green fluorescent protein chimeric mouse model. Biochem Biophys Res Commun 331: 31-36.

Toupadakis CA, Wong A, Genetos DC, Chung DJ, Murugesh D, Anderson MJ, Loots GG, Christiansen BA, Kapatkin AS, Yellowley CE (2012) Long-term administration of AMD3100, an antagonist of SDF-1/ CXCR4 signaling, alters fracture repair. J Orthop Res 30: 1853-1859.

Editor's note: All of the questions raised by the reviewers were addressed by the authors in the revised manuscript accepted for publication. Therefore, there is no Discussion with Reviewers section for this paper. 